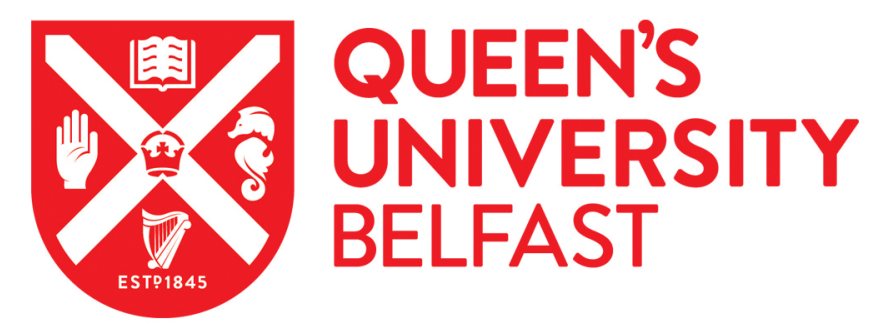

\title{
Non-equilibrium properties of trapped ions under sudden application of a laser
}

Cifuentes, A. A., Nicacio, F., Paternostro, M., \& Semiao, F. L. (2016). Non-equilibrium properties of trapped ions under sudden application of a laser. Physical Review A (Atomic, Molecular, and Optical Physics), 94(1), [013406]. https://doi.org/10.1103/PhysRevA.94.013406

Published in:

Physical Review A (Atomic, Molecular, and Optical Physics)

Document Version:

Peer reviewed version

Queen's University Belfast - Research Portal:

Link to publication record in Queen's University Belfast Research Portal

Publisher rights

@2016 American Physical Society.

\section{General rights}

Copyright for the publications made accessible via the Queen's University Belfast Research Portal is retained by the author(s) and / or other copyright owners and it is a condition of accessing these publications that users recognise and abide by the legal requirements associated with these rights.

Take down policy

The Research Portal is Queen's institutional repository that provides access to Queen's research output. Every effort has been made to ensure that content in the Research Portal does not infringe any person's rights, or applicable UK laws. If you discover content in the Research Portal that you believe breaches copyright or violates any law, please contact openaccess@qub.ac.uk. 


\title{
Non-equilibrium properties of trapped ions under sudden application of a laser
}

\author{
A. A. Cifuentes, ${ }^{1}$ F. Nicacio, ${ }^{1}$ M. Paternostro, ${ }^{2}$ and F. L. Semião ${ }^{1}$ \\ ${ }^{1}$ Centro de Ciências Naturais e Humanas, Universidade Federal do ABC, 09210-170, Santo André, São Paulo, Brazil \\ ${ }^{2}$ Centre for Theoretical Atomic, Molecular, and Optical Physics, \\ School of Mathematics and Physics, Queens University, Belfast BT7 1NN, United Kingdom
}

\begin{abstract}
Coherent quantum-state manipulation of trapped ions using classical laser fields is a trademark of modern quantum technologies. In this work, we study aspects of work statistics and irreversibility in a single trapped ion due to sudden interaction with the impinging laser. This is clearly an out-of-equilibrium process where work is performed through illumination of an ion by the laser. Starting with the explicit evaluation of the first moments of the work distribution, we proceed to a careful analysis of irreversibility as quantified by the nonequilibrium lag. The treatment employed here is not restricted to the Lamb-Dicke limit, what allows us to investigate the interplay between nonlinearities and irreversibility. We show, for instance, that in the resolved carrier and sideband regimes, variation of the Lamb-Dicke parameter may cause a non-monotonic behavior of the irreversibility indicator. Counterintuitively, we find a working point where nonlinearity helps reversibility, making the sudden quench of the Hamiltonian closer to what would have been obtained quasistatically and isothermally.
\end{abstract}

\section{INTRODUCTION}

Quantum control is key to quantum technologies [1]. Trapping of neutral or charged particles, assisted by cooling techniques to bring them to ultracold temperatures, form a mature experimental platform to the development of quantum control. In particular, laser-manipulated trapped ions are now one of the most developed settings for experimental investigation of quantum effects and the implementation of basic building blocks needed for quantum storage, communication and processing of information [2-5]. Along with the development of quantum technologies, where one is usually interested in situations far from thermal equilibrium to fully harness the power of quantum coherences, there has been an increasing interest in non-equilibrium thermodynamics of quantum systems, sometimes referred to as Quantum Thermodynamics (QT). Properly setting the limits in the extraction of useful work and the related problem of entropy production in non-equilibrium processes in quantum systems lie at the core of QT [6-8]. Despite being a relatively new subject, QT is a field which grows steadily and rapidly [9-16]. QT brings the old realm of classical thermodynamics to a new perspective where quantum correlations and quantum coherence might play an important role.

Taking all this into account, it seems crucial to investigate the interaction of laser fields and trapped ions from a statistical non-equilibrium perspective, trying to uncover new aspects and physically relevant information previously untouched by standard approaches to this subject. The scenario is particularly rich given the multitude of different energy structures and transitions involving electronic and vibrational degrees of freedom accessible in this system through laser interaction [2, 17]. One of the motivations for the present work is the possibility of studying thermodynamical implications of nonequivalent physical regimes, some of them driven by strongly nonlinear Hamiltonians [2, 17]. The interplay between the physics of trapped ions and QT has been explored previously, for instance, in the context of ion-based thermoengines [18] and the verification of fluctuation relations [19].
However, our work departs from those in both context and methodology. In particular, we bring the variety of physical regimes available in the trapped ion system to the light of nonequilibrium QT. This includes carrier and sideband regimes, where single- or multiple-quanta transitions and energy dependent couplings manifest according to the laser frequency [2]. We will be particularly interested in a study that addresses thermodynamic irreversibility as quantified by the irreversible lag [20] produced by a finite-time transformation experienced by a trapped ion.

This paper is organized as follows. In Section II, we briefly review the relevant concepts of non-equilibrium QT needed to the subsequent developments. This includes a discussion of the microscopic view of work and the basic physics upon which the nonequilibrium lag is built. Sec. III is dedicated to a brief review of the laser-manipulated trapped ion system. Our results are presented in Section IV, where we discuss work and irreversibility when the trapped ion is suddenly shinned by a classical laser field. In Section V, we conclude our findings and, in the Appendix, we present expressions for the eigenvalues and eigenvectors of the system Hamiltonian for an arbitrary sideband.

\section{WORK AND IRREVERSIBILITY}

A non isolated system can exchange energy and/or particles with the environment or an external agent. In this context, work is energy which is transferred/extracted to/from the system through application of arbitrary generalized forces [21]. From a microscopic perspective, work is then necessarily accompanied by a modification of the system Hamiltonian (energy levels). This is to be distinguished from heat which is energy exchanged with the environment through their mutual weak (infinitesimal) interaction. Consequently, the effect of heat is not a significant modification of the energy levels but a redistribution of their population. This too corresponds to a variation of internal energy just like work.

In general, a non-isolated system may suffer both processes. However, in what follows, we will be interested in 
the scenario where work is performed without heat exchange. This can be physically achieved, for instance, when the system is thermally isolated or the work protocol is performed in a time interval which is orders of magnitude shorter than the thermalization time. This is precisely the case of the idealized process of sudden Hamiltonian quench which corresponds to the instantaneous change of the system Hamiltonian from $\hat{\mathcal{H}}\left(\lambda_{i}\right)$ to $\hat{\mathcal{H}}\left(\lambda_{f}\right)$. In these expressions, $\lambda$ is a macroscopic variable in the system Hamiltonian usually called work parameter in the context of non-equilibrium thermodynamics. Work $W$ is a random variable encompassing both thermal and quantum fluctuations. In the case of a sudden quench, the statistical moments of the work distribution read [22]

$$
\left\langle W^{n}\right\rangle=\sum_{k=0}^{n}(-1)^{k}\left(\begin{array}{l}
n \\
k
\end{array}\right) \operatorname{Tr}\left[\hat{\mathcal{H}}_{f}^{(n-k)} \hat{\mathcal{H}}_{i}^{k} \hat{\rho}_{i}\right]
$$

with $n$ integer and $\hat{\mathcal{H}}_{j}:=\hat{\mathcal{H}}\left(\lambda_{j}\right)$ for $j=i, f$. More details about the statistical meaning of work can be found in [23] and references therein.

One of the most important results of non-equilibrium statistical mechanics is the Jarzinsky equality [9], from which one can directly obtain a fundamental inequality involving average work $\langle W\rangle$ and Helmholtz free energy $F$

$$
\langle W\rangle \geq \Delta F
$$

where $\Delta F \equiv F\left(\lambda_{f}, \beta\right)-F\left(\lambda_{i}, \beta\right)$ is the difference between the free energies of the system. Explicitly,

$$
F\left(\lambda_{j}, \beta\right)=-\frac{1}{\beta} \ln \mathcal{Z}\left(\lambda_{j}\right), \quad \mathcal{Z}\left(\lambda_{j}\right) \equiv \operatorname{Tr} \mathrm{e}^{-\beta \hat{\mathcal{H}}\left(\lambda_{j}\right)},
$$

with $j=i, f$. The equality in Eq. (2) is only achieved by an isothermal quasistatic process, which is reversible [23].

The indicator of irreversibility used in this work can then be defined considering what has just been exposed. Based on Eq. (2), one defines [10, 20]

$$
\mathcal{L} \equiv \beta(\langle W\rangle-\Delta F)
$$

as an indicator of irreversibility in the sense that the work protocol is reversible only when $\mathcal{L}=0$. What is reversible or irreversible for this indicator is the work protocol realized in a initially equilibrated system. The idea is that a backwards run of the work protocol after the system starts attempting thermal reequilibration will not, in general, bring the system and environment to their initial state. The quantity between the parenthesis is known as irreversible work [10], and $\mathcal{L}$ is usually called "nonequilibrium lag" (NL) as it gives an idea of how the system state, after the work protocol, lags behind an equilibrium thermal state fixed by the final Hamiltonian and inverse temperature $\beta$. Remarkably, it has been show that the NL is exactly equal to the relative entropy between the thermal state used to evaluate $F\left(\lambda_{f}, \beta\right)$ and the post-work state [20]. It is important to remark that the relative entropy is zero for identical states and it diverges for orthogonal states [24].

\section{SHORT REVIEW ON TRAPPED IONS INTERACTING WITH CLASSICAL LASER FIELDS}

We now present the basic elements needed to work with trapped ions subjected to laser fields. More information can be found in the many reviews available in the literature, e.g., [2]. Usually, the laser-ion setup is described by a model consisting of a two-level system (electronic degrees of freedom) coupled to a harmonic oscillator (center of mass motion). The latter is the result of electromagnetic confinement achieved by the use of trapping technology, e.g., Paul traps [25], and the electronic-motion coupling occurs due to momentum exchange with the laser.

By considering the center of mass (CM) degree of freedom as an oscillator with natural frequency $\nu$, and the two levels $\{|g\rangle,|e\rangle\}$ with an energy separation of $\hbar \omega_{0}$, the system Hamiltonian reads [26]

$$
\hat{\mathcal{H}}=\hat{\mathcal{H}}_{0}+\hat{\mathcal{H}}_{\mathrm{I}}
$$

with

$$
\hat{\mathcal{H}}_{0}=\hbar \nu \hat{a}^{\dagger} \hat{a}+\frac{\hbar \omega_{0}}{2} \hat{\sigma}_{z}
$$

and

$$
\hat{\mathcal{H}}_{\mathrm{I}}=\frac{\hbar \Omega}{2}\left[\hat{\sigma}_{+} \mathrm{e}^{i \eta\left(\hat{a}+\hat{a}^{\dagger}\right)-i \omega_{L} t}+\hat{\sigma}_{-} \mathrm{e}^{-i \eta\left(\hat{a}+\hat{a}^{\dagger}\right)+i \omega_{L} t}\right]
$$

where $\omega_{L}$ is the laser frequency, $\Omega$ the classical Rabi frequency, $\hat{a}$ the annihilation operator for the CM motion, $\hat{\sigma}_{z}=$ $|e\rangle\langle e|-| g\rangle\left\langle g\left|, \hat{\sigma}_{+}=\hat{\sigma}_{-}^{\dagger}=\right| e\right\rangle\langle g|$, and $\eta$ the Lamb-Dicke parameter defined as

$$
\eta=\frac{\omega_{L}}{c} \sqrt{\frac{\hbar}{2 M \nu}} \cos \phi,
$$

with $M$ being the mass of the trapped ion, $c$ the speed of light, and $\phi$ the angle between the laser wave vector and the trap axis (one dimensional motion).

Depending on the detuning $\omega_{0}-\omega_{L}$, the laser will cause the coupling of different vibrational levels with electronic part, each case representing a different quantum-optical process [27] with its own effective Hamiltonian. The procedure to reveal each of those Hamiltonians is very well described in the literature, e.g., [2, 17]. Basically, after setting $\omega_{0}-\omega_{L}= \pm m \nu$, with $m=0,1,2, \ldots$, one applies a rotating wave approximation (RWA) to Hamiltonian (5) in order to obtain

$$
\hat{\mathcal{H}}_{ \pm}^{(m)}=\hat{\mathcal{H}}_{0}+\hbar\left(\mathrm{e}^{-i \omega_{L} t} \hat{\Omega}_{m}^{ \pm} \hat{\sigma}_{+}+\mathrm{e}^{i \omega_{L} t} \hat{\Omega}_{m}^{\mp} \hat{\sigma}_{-}\right)
$$

where

$$
\hat{\Omega}_{m}^{+}=\hat{\Omega}_{m}^{-\dagger}=\frac{\Omega}{2} \mathrm{e}^{-\eta^{2} / 2} \sum_{l=0}^{\infty}(i \eta)^{2 l+m} \frac{\hat{a}^{\dagger l} \hat{a}^{l}}{l !(l+m) !} \hat{a}^{m} .
$$

For consistence, one must notice that $\eta$ in Eq. (8), besides being a function of $\phi$ and $\nu$, is also a function of $\omega_{0}$. This is 
so because $\omega_{L}$ is now fixed by the sideband choice (value of $m)$.

The Hamiltonian $\hat{\mathcal{H}}_{+}^{(m)}$ is obtained with $\omega_{0}-\omega_{L}=m \nu$, and it describes a $m$-phonon process for the vibrational part accompanied with transitions in the atomic levels. It can be referred to as a $m$-phonon Jaynes-Cummings (JC) model. On the other hand, Hamiltonian $\hat{\mathcal{H}}_{-}^{(m)}$ is obtained with $\omega_{0}-\omega_{L}=$ $-m \nu$ and it can be referred to as a $m$-phonon anti-JaynesCummings (AJC) model. The case $m=0$ can be studied using either $\hat{\mathcal{H}}_{+}^{(m)}$ or $\hat{\mathcal{H}}_{-}^{(m)}$,

$$
\hat{\mathcal{H}}^{(0)}=\hat{\mathcal{H}}_{ \pm}^{(0)}=\hat{\mathcal{H}}_{0}+\frac{\hbar}{2}\left(\mathrm{e}^{-i \omega_{L} t} \hat{\Omega}_{0}^{+} \hat{\sigma}_{+}+\mathrm{e}^{i \omega_{L} t} \hat{\Omega}_{0}^{-} \hat{\sigma}_{-}\right),
$$

and it describes Rabi oscillations between electronic levels, i.e., the carrier transitions $[2,17]$.

For what comes next, it is useful to present now the matrix elements of $\hat{\Omega}_{m}^{ \pm}$in the Fock basis of the CM harmonic motion

$$
\begin{aligned}
\left\langle n\left|\hat{\Omega}_{m}^{+}\right| n^{\prime}\right\rangle & =\left\langle n^{\prime}\left|\hat{\Omega}_{m}^{-}\right| n\right\rangle^{*} \\
& =\frac{\Omega(i \eta)^{m}}{2} \sqrt{\frac{n !}{(m+n) !}} \mathrm{e}^{-\eta^{2} / 2} L_{n}^{m}\left(\eta^{2}\right) \delta_{n^{\prime} n+m},
\end{aligned}
$$

with the associated Laguerre polynomials [28]

$$
L_{n}^{m}(x)=\sum_{k=0}^{n}(-1)^{k} \frac{(n+m) !}{(m+k) !(n-k) !} \frac{x^{k}}{k !} .
$$

As it can be seen from Eq. (12), the quantum Rabi frequencies, $\left\langle n\left|\hat{\Omega}_{m}^{+}\right| n^{\prime}\right\rangle$, have a strong dependence on the LambDicke parameter $\eta$. For small values of $\eta$, they present a quasilinear dependence on $n$, typical of $m$-photon JaynesCummings models in the context of cavity quantum electrodynamics (cQED) [29]. However, for the ionic system, it is possible to induce considerable nonlinearities in $\left\langle n\left|\hat{\Omega}_{m}^{+}\right| n^{\prime}\right\rangle$ simply by increasing the Lamb-Dicke parameter. The quantum Rabi frequencies become a oscillating function of $n$ due to the presence of the Laguerre polynomials in Eq. (12). These oscillations can strongly influence the system dynamics as thoroughly studied in [30].

\section{RESULTS}

The work protocol we have in mind is now explained. First, the work parameter $\lambda_{t}$ here has to do with the application of the laser on the ion. More specifically, we take $\lambda_{i}=0$ and $\lambda_{f}=\Omega$ in a sudden quench of the system Hamiltonian. This means an abrupt change from

$$
\hat{\mathcal{H}}\left(\lambda_{i}\right)=\hbar \nu \hat{a}^{\dagger} \hat{a}+\frac{\hbar \omega_{0}}{2} \hat{\sigma}_{z}
$$

to

$$
\hat{\mathcal{H}}\left(\lambda_{f}\right)=\hat{\mathcal{H}}\left(\lambda_{i}\right)+\frac{\hbar \Omega}{2}\left[\hat{\sigma}_{+} \mathrm{e}^{i \eta\left(\hat{a}+\hat{a}^{\dagger}\right)}+\hat{\sigma}_{-} \mathrm{e}^{-i \eta\left(\hat{a}+\hat{a}^{\dagger}\right)}\right],
$$

or, if we want to explore the sidebands, an abrupt change to

$$
\hat{\mathcal{H}}\left(\lambda_{f}\right)=\hat{\mathcal{H}}\left(\lambda_{i}\right)+\hbar\left(\hat{\Omega}_{m}^{ \pm} \hat{\sigma}_{+}+\hat{\Omega}_{m}^{\mp} \hat{\sigma}_{-}\right) .
$$

The above Hamiltonians, Eq. (15) and Eq. (16), correspond to the sudden application of the laser field, i.e., the result of taking the limit of $t \rightarrow 0$ in Eq. (7) and in Eq. (9), respectively.

It is well known that the Hamiltonian (15) can not be diagonalized exactly, so that much of the analytical advances take place with the sideband Hamiltonians in Eq. (16). It is important to remark that Eq. (16) indeed describe quite well the system when $\omega_{0}-\omega_{L}= \pm m \nu$ and $\Omega$ is moderately week, which are conditions easily implemented in the laboratories $[4,5]$. Before the interaction with the laser, the trapped ion is found to be in thermal equilibrium with the environment (at inverse temperature $\beta$ ). This is described by the Gibbs state associated with Hamiltonian Eq. (14), i.e.,

$$
\hat{\rho}_{i}=\frac{\mathrm{e}^{-\beta \hbar \nu \hat{n}}}{(\bar{n}+1)} \otimes \frac{\mathrm{e}^{-\frac{\beta \hbar \omega_{0}}{2} \hat{\sigma}_{z}}}{2 \cosh \frac{\beta \hbar \omega_{0}}{2}},
$$

where $\hat{n}=\hat{a}^{\dagger} \hat{a}$ is the number operator and

$$
\bar{n}=\operatorname{Tr}\left(\hat{n} \hat{\rho}_{i}\right)=\left(\mathrm{e}^{\beta \hbar \nu}-1\right)^{-1}
$$

is the thermal occupation number of the CM motion.

In spite of the difficulties found in dealing with the full Hamiltonian Eq. (15), we were able to find the first moments of the work distribution. This is already valuable information because to obtain the full distribution we would need the whole set of eigenvalues and eigenvectors of Eq. (15) which are not possible to be obtained, except numerically and to a restrict precision giving the complexity of the Hamiltonian. We then use Eq. (1), appropriate to a sudden change, to calculate a few first moments of the work distribution and get some insight of it.

The first moment, $n=1$, using Eq. (15) and Eq. (17), turns out to be

$$
\langle W\rangle=\operatorname{Tr}\left[\hat{\mathcal{H}}_{\mathrm{I}} \hat{\rho}_{i}\right] \propto \operatorname{Tr}\left[\hat{\sigma}_{ \pm} \mathrm{e}^{-\frac{\beta \hbar \omega_{0}}{2} \hat{\sigma}_{z}}\right]=0 .
$$

As for the second, we now find

$$
\left\langle W^{2}\right\rangle=\hbar^{2} \Omega^{2} / 4,
$$

which, interesting enough, depends only on the magnitude of the work parameter $\Omega$ (controlled by laser power) and it is completely independent of the temperature. Since $\langle W\rangle=0$, the second moment is also the variance of the work distribution. The third moment is given by

$$
\left\langle W^{3}\right\rangle=\frac{\hbar^{3} \Omega^{2}}{4}\left[\nu \eta^{2}+\omega_{0} \tanh \frac{\beta \hbar \omega_{0}}{2}\right],
$$

in which appears the dependence on the temperature. From the second and third moments, we can determine the skewness of the work distribution $\left\langle W^{3}\right\rangle /\left\langle W^{2}\right\rangle^{3 / 2}$. This turns out to be inversely proportional to the magnitude of the work parameter. Consequently, the stronger the laser, the more symmetric the distribution is around the mean value $\langle W\rangle=0$. Since $\left\langle W^{3}\right\rangle>0$, as seen from Eq. (21), the work distribution is biased towards negative values of work. All these facts about the first moments of the work distribution, obtained with the 
full Hamiltonian Eq. (15), tell us that negative work (internal energy descrease) is more likely than the equivalent positive work (internal energy increase) at the very first instant of interaction with the laser field. Note also that the asymmetry around the mean value decreases with the temperature while increases with $\eta$. Finally, according to Eq. (8), $\left\langle W^{3}\right\rangle$ and the skewness are actually independent of the trap frequency $\nu$.

Now we turn our attention to the sideband Hamiltonians in Eq. (16) and to the irreversibility of the work protocol consisting of the sudden quench of system Hamiltonian due to laser interaction. As said before, these effective Hamiltonians are obtained from the full Hamiltonian Eq. (15) by setting resonance $\omega_{0}=\omega_{L} \pm m \nu$ and performing a rotating wave approximation. We will see that a thermodynamic analysis is able to reveal the different aspects of the optical processes raised by the selection of distinct sidebands.

We proceed to apply the NL in Eq. (4) to reveal the irreversibility of the work protocol. Just like happened with the full Hamiltonian (15), the first moment of the work distribution or simply the average work is again null, i.e., $\langle W\rangle=0$. For this reason, the NL in Eq. (4) for the sudden quench of the sideband Hamiltonian in Eq. (16) reads

$$
\mathcal{L}=\ln \frac{\mathcal{Z}\left(\lambda_{f}\right)}{\mathcal{Z}\left(\lambda_{i}\right)}
$$

with

$$
\mathcal{Z}\left(\lambda_{i}\right)=2(\bar{n}+1) \cosh \frac{\beta \hbar \omega_{0}}{2},
$$

obtained using Eq. (14), and

$$
\mathcal{Z}_{ \pm}\left(\lambda_{f}\right)=\sum_{n=0}^{\infty}\left[\mathrm{e}^{-\beta \mu_{ \pm}^{(n, m)}}+\mathrm{e}^{-\beta \gamma_{ \pm}^{(n, m)}}\right]+\sum_{n=0}^{m-1} \mathrm{e}^{-\beta \zeta_{ \pm}^{(n, m)}}
$$

obtained with Eq. (16). The functions $\mu_{ \pm}, \gamma_{ \pm}$, and $\zeta_{ \pm}$are the eigenvalues of the Hamiltonians in (16), and their expressions can be found in Eqs. (A-4), (A-5), (A-9), and (A-10), what allows one to get

$$
\begin{aligned}
\mathcal{Z}_{ \pm}\left(\lambda_{f}\right)= & 2 \sum_{n=0}^{\infty} \mathrm{e}^{-\beta \hbar \nu\left(n+\frac{m}{2}\right)} \cosh \left[\frac{\beta \hbar}{2} \sqrt{\omega_{L}^{2}+\Omega^{2}\left|f_{n}^{m}\right|^{2}}\right] \\
& +(\bar{n}+1)\left(1-\mathrm{e}^{-\beta \hbar m \nu}\right) \mathrm{e}^{ \pm \frac{1}{2} \beta \hbar \omega_{0}}
\end{aligned}
$$

with $\omega_{L}=\omega_{0} \mp m \nu$, and

$$
f_{n}^{m}:=\frac{2}{\Omega}\left\langle n\left|\hat{\Omega}_{m}^{+}\right| n+m\right\rangle=(i \eta)^{m} \sqrt{\frac{n !}{(m+n) !}} \mathrm{e}^{-\frac{\eta}{2} 2} L_{n}^{m}\left(\eta^{2}\right)(26)
$$

where we used Eq. (12). With the above two partition functions, we can calculate $\mathcal{L}$ in Eq. (22). Before the presentation of the simulations, we want to make notation clear emphasizing that $\mathcal{Z}_{+}\left(\lambda_{f}\right)$ refers the JC-type Hamiltonians with $\omega_{L}=\omega_{0}-m \nu$, while $\mathcal{Z}_{-}\left(\lambda_{f}\right)$ refers to the AJC-type Hamiltonians with $\omega_{L}=\omega_{0}+m \nu$.

Now we carry on to the numerical investigation of the NL. For that, it is important to have in mind the reality of the physical parameters to be used in the simulations. First, the initial thermal occupation numbers $\bar{n}$ will be considered relatively small in order to have quantum fluctuations playing some role. The experiments employ sophisticated and very eficient cooling techniques for that aim [2]. For the typical frequencies and coupling constants, we will be focusing on the experimental implementation of Eq. (9) using $\mathrm{Ca}^{+}$ions [4]. In these experiments, the electronic level separation is about $\mathrm{THz}$ while the trap frequencies are set typically in some $\mathrm{MHz}$, and one order of magnitude smaller or higher by adjusting the trap potentials. For the classical Rabi frequency $\Omega$, a few $\mathrm{MHz}$ is also a realistic choice. We would also like to emphasize that our analysis and results are suitable to be applied to other known experimental setups such as those involving $\mathrm{Be}^{+}[5]$ or $\mathrm{Yb}^{+}$ [31].

The dependence of NL on the Lamb-Dicke parameter $\eta$ is presented in Fig. 1 for a few values of $m$. The variation of $\eta$ in these plots comes from $\phi$ in Eq. (8), since we are keeping the trap and laser frequencies fixed. For small $\eta$, i.e., in the Lamb-Dicke regime, the Hamiltonians $\hat{\mathcal{H}}_{ \pm}^{(m)}$ are basically ordinary Jaynes-Cummings models from cQED, in the sense that Eq. (10) becomes approximately independent of the energy or number operator $\hat{a}^{\dagger} \hat{a}$. In this regime, both the JC and AJC cases present the same ordering with respect to $m$. We see that the higher the sideband, or the number of motional quanta absorbed in the transition driven by the laser, the lesser the lag is. This means that the sudden application of the laser becomes less irreversible and more like a quasistatic change. However, by increasing non-linearity, i.e., the magnitude of $\eta$, we depart from the ordinary cQED models, and Fig. 1 reveals that the JC and AJC present different responses with respect to irreversibility. For the JC case, increasing $\eta$ do not alter the order with respect to $m$ and, the higher the sideband, the lesser the NL. On the other hand, for the AJC such an order is not respected and, interesting enough, it comes to a point in which the higher the sideband, the higher the NL. Such behavior is induced by nonlinearity and it highlights well the different thermodynamic aspects resulting from JC and AJC models using trapped ions.

The behavior of the NL as $\eta$ is varied, with $\nu, \omega_{0}$ kept fixed, is determined by $f_{n}^{m}$ defined in Eq. (26). In order to gain some insight about what was seen numerically in Fig. 1, we now resort to analytical asymptotic limits. For large $\eta$, the
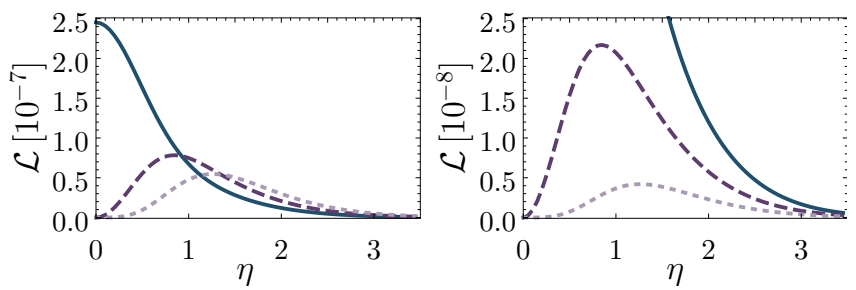

Figure 1. (Color Online) NL as a function of the Lamb-Dicke parameter. The AJC (left) and JC (right) cases presented for $m=0$ (solid), $m=1$ (dashed) and $m=2$ (dotted). The solid line is the same line in both panels. The used physical parameters were $\Omega=$ $1.0 \pi \mathrm{MHz}, \omega_{0}=822.0 \pi \mathrm{THz}, \nu=5.0 \mathrm{KHz}, M=7.0 \times 10^{-26} \mathrm{~kg}$, and $\bar{n}=0.38$. Eq. (25) is evaluated using the first 40 terms in the summation [32]. 
function $\left|f_{n}^{m}\right| \rightarrow 0$ because of the exponential in Eq. (26). In this limit, $\mathcal{Z}_{ \pm}\left(\lambda_{f}\right) \rightarrow \mathcal{Z}\left(\lambda_{i}\right)$ so that $\mathcal{L} \rightarrow 0$.

In the Lamb-Dicke regime, $\eta \ll 1$, we expand the exponential and Laguerre in Eq. (26) up to second order in $\eta$ to find

$$
\left|f_{n}^{m}\right|^{2} \approx \frac{(n+m) !}{n ! m !^{2}}\left[1-\frac{2 n+m+1}{m+1} \eta^{2}\right] \eta^{2 m}
$$

To obtain this expression we used $d L_{n}^{m}(x) / d x=-L_{n-1}^{m+1}(x)$ and $L_{n}^{m}(0)=(n+m) ! /(n ! m !)$ [28]. Now, by keeping just terms up to $\eta^{2}$ in $\left|f_{n}^{m}\right|^{2}$, one gets

$$
\left|f_{n}^{m}\right|^{2} \approx\left[1-(2 n+1) \eta^{2}\right] \delta_{m 0}+(n+1) \eta^{2} \delta_{m 1} .
$$

Terms with $m \geq 2$ appear only in higher powers of $\eta$. Notice that for $m=1,\left|f_{n}^{1}\right|^{2} \rightarrow 0$ and $\mathcal{Z}_{ \pm}\left(\lambda_{f}\right) \rightarrow \mathcal{Z}\left(\lambda_{i}\right)$ as $\eta \rightarrow 0$, what makes $\mathcal{L} \rightarrow 0$. On the other hand, $\left|f_{n}^{0}\right|^{2}$ in Eq. (28) is a concave function of $\eta$ with $\lim _{\eta \rightarrow 0}\left|f_{n}^{0}\right|^{2}=1, \forall n$. Consequently, $\mathcal{L} \neq 0$ as $\eta \rightarrow 0$. All these features can be seen from Fig. 1. For $m>1$, only higher order terms in $\eta$ contribute to $\left|f_{n}^{m}\right|$, forcing $\mathcal{Z}_{ \pm}\left(\lambda_{f}\right) \rightarrow \mathcal{Z}\left(\lambda_{i}\right)$ as $\eta \rightarrow 0$, just like happens when $m=1$.

The physical explanation for the distinct behavior found in the carrier transition $m=0$ lies in the system Hamiltonian after and before laser application. From Eq. (10), one can see that

$$
\lim _{\eta \rightarrow 0} \hat{\Omega}_{m}^{ \pm}=\frac{\Omega}{2} \delta_{m 0} \mathbb{1},
$$

where $\mathbb{1}$ is the identity operator for the center-of-mass motion. By taking Eqs. (11) and (29) into account, it follows that, when $m=0$, the laser is able to drive transitions between the two electronic states, even when $\eta=0$. In other words, the pre- and post-quench Hamiltonians are different in the limit $\eta \rightarrow 0$, only when $m=0$. The process becomes then reversible in such a limit, provided $m \neq 0$.

Now, we investigate the role of the classical Rabi frequency $\Omega$ on the irreversibility. The result is depicted in Fig. 2, where one can see that the NL increases with $\Omega$. This behavior is expected from the detailed analysis of Eq. (25), and it can be physically understood from the fact that $\Omega$ is the work parameter and quantifies the intensity of the sudden quench.
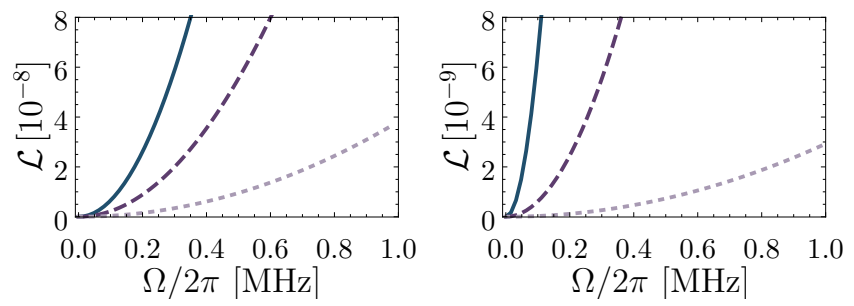

Figure 2. (Color Online) Nonequilibrium Lag as a function of the Rabi Frequency of the AJC case (left) and JC case (right). We use $\eta=0.5$ and everything else is the same as in Fig. 1.

In order to obtain a better understanding of the problem, it is necessary to go on and investigate the role of temperature. The NL as a function of the mean occupation number
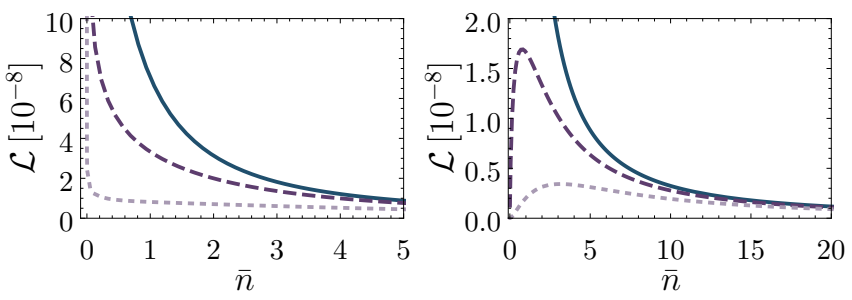

Figure 3. (Color Online) Nonequilibrium Lag as a function of the mean occupation number of the initial thermal state for the AJC case (left) and for JC case (right). We have used $\eta=0.5$ and everything else is the as in Fig. 1, except for the sum in Eq. (25) which is now evaluated with $n=2000$ for the plot on the left and $n=5000$ for the one on the right [32].

of the initial thermal state $\bar{n}$ in Eq. (18) is presented in Fig. 3. It is noticeable that the AJC and JC models in the trapped ion system may respond so differently to variations of initial thermal energy of the system. In particular, it can be seen from Fig. 3 that the shown sidebands for the AJC and also for $m=0$ (which can be seen as either belonging to the AJC or JC classes) lead to a divergency in the NL as $\bar{n} \rightarrow 0(\beta \rightarrow \infty)$. This is not observed for for the JC case.

Although the dependence of $\mathcal{L}$ on the temperature is a bit more intricate, since all factors in Eq. (25) depend on it, we again succeeded in providing an analytical treatment based on asymptotics that helps us to spot the reasons behind such different behavior found in the JC and AJC models. In the high temperature limit $\beta \rightarrow 0(\bar{n} \rightarrow \infty)$, a successive application of this limit, first to some exponentials and then to the hyperbolic functions in Eq. (25), results in

$$
\frac{\mathcal{Z}_{ \pm}\left(\lambda_{f}\right)}{\mathcal{Z}\left(\lambda_{i}\right)} \rightarrow \lim _{\beta \rightarrow 0}\left[(\bar{n}+1)^{-1} \sum_{n=0}^{\infty} \mathrm{e}^{-\beta \hbar \nu\left(n+\frac{m}{2}\right)}\right]=1,
$$

what makes $\mathcal{L} \rightarrow 0$. This shows that, in this limit, the dynamics becomes reversible regardless of $m$.

For low temperatures, one can write $\cosh \beta x \approx \frac{1}{2} \mathrm{e}^{\beta|x|}$ to find

$$
\frac{\mathcal{Z}_{ \pm}\left(\lambda_{f}\right)}{\mathcal{Z}\left(\lambda_{i}\right)} \rightarrow \lim _{\beta \rightarrow \infty}\left[\left(1-\delta_{m 0}\right) \mathrm{e}^{-\frac{\beta \hbar \omega_{0}(1 \mp 1)}{2}}+\sum_{n=0}^{\infty} \mathrm{e}^{-\frac{\beta \hbar}{2} \Phi_{n}^{m}}\right],
$$

where we have defined

$$
\Phi_{n}^{m}:=\nu(2 n+m)+\omega_{0}-\sqrt{\left(\omega_{0} \mp m \nu\right)^{2}+\Omega^{2}\left|f_{n}^{m}\right|^{2}} .
$$

From this, we can analyze individually the AJC and JC cases. For the AJC and the carrier $m=0$, it is easy to see that $\Phi_{0}^{m}<$ $0, \forall m$. As a result,

$$
\lim _{\beta \rightarrow \infty} \frac{\mathcal{Z}_{-}\left(\lambda_{f}\right)}{\mathcal{Z}\left(\lambda_{i}\right)}=\infty, \forall m
$$

showing that for $\hat{\mathcal{H}}_{-}^{(m)}$ in Eq. (9) and $\hat{\mathcal{H}}^{(0)}$ in Eq. (11) the NL Eq. (22) always diverges when $\beta \rightarrow \infty$. For the JC case, we must give a closer look at the function $\Phi_{n}^{m}$. From Eq. (31), 
and remembering that the case $m=0$ was already analyzed in Eq. (33),

$$
\frac{\mathcal{Z}_{+}\left(\lambda_{f}\right)}{\mathcal{Z}\left(\lambda_{i}\right)} \rightarrow 1+\lim _{\beta \rightarrow \infty} \sum_{n=0}^{\infty} \mathrm{e}^{-\frac{\beta \hbar}{2} \Phi_{n}^{m}}
$$

If, for a given $m$, at least one of the $\Phi_{n}^{m}$ appearing in Eq. (34) is negative, the above limit diverges and $\mathcal{L} \rightarrow \infty$. On the other hand, provided $\Phi_{n}^{m} \geq 0$ for all $n$, then

$$
\frac{\mathcal{Z}_{+}\left(\lambda_{f}\right)}{\mathcal{Z}\left(\lambda_{i}\right)} \rightarrow k+1
$$

where $k$ is the number of times $\Phi_{n}^{m}$ equals zero. Consequently, $\mathcal{L} \rightarrow \ln (1+k)$ for the JC case. For the parameters chosen in Fig. 3, the JC case corresponds to $\Phi_{n}^{m} \geq 0$ and the limit in Eq. (35) holds with $k=0$, i.e., no divergence is observed.

Divergences of the NL can be understood, in general, as a consequence of the distinguishability between the post-work state and the reference thermal state used to evaluate the final free energy $F\left(\lambda_{f}, \beta\right)$. As previously commented, the NL can be written in terms of the relative entropy between those two states [20]. As so, the smaller the NL, the more indistinguishable the two states are and, for orthogonal states, it diverges. In a quench process, as considered here, the initial state does not change after the work protocol [22]. Consequently, the post-work state is a Gibbs state defined with inverse temperature $\beta$ and Hamiltonian (14). When $\beta \rightarrow \infty$, this state is basically $|0, g\rangle$. In the same limit, the reference thermal state used to evaluate $F\left(\lambda_{f}, \beta\right)$ will be given by the ground state of either the JC Hamiltonian or the AJC Hamiltonian, depending on the chosen $\omega_{L}$. For the physical parameters used in the simulations, the ground state of the JC Hamiltonian coincides with the post-work state which is $|0, g\rangle$, while the ground state of the AJC Hamiltonian will be a superposition of $|0, e\rangle$ and $|m, g\rangle$. We can then see that NL will be smaller for the JC than for the AJC because the post-work state is more indistinguishable from the ground state of the former than from the ground state of the latter. All eigenstates and eigenvalues for AJC and JC Hamiltonians can be found in the appendix.

We may wonder under which parameters choice the JC case can present divergences in the NL. In order words, how the system parameters can be chosen to cause at least one of the $\Phi_{n}^{m}$ in (34) to be negative. The analysis of Eq. (32) reveals that this is the case provided

$$
\left|f_{n}^{m}\right|>\frac{2}{\Omega} \sqrt{\nu\left(\omega_{0}+n \nu\right)(n+m)}
$$

for a fixed $m$ (sideband) and some value of $n$. Now, in order to see this effect, one needs to go a bit beyond the current experimental set of parameters found in the literature. The result is shown in Fig. 4 where the parameters were deliberately chosen as to imply $\Phi_{n}^{m}<0$ in some of the examples, making Eq. (35) invalid and causing the NL to diverge as $\beta \rightarrow \infty$.

We now discuss the dependence of the Lamb-Dicke parameter on the trap frequency $\nu$ in Eq. (8) and its implication for the irreversibility of the process. For that, we consider as one
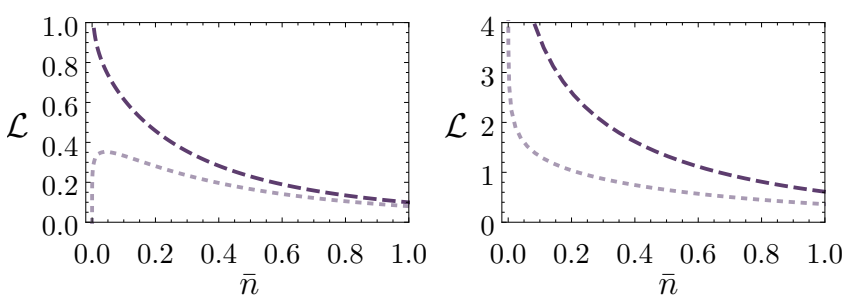

Figure 4. (Color Online) Nonequilibrium Lag as a function of the mean occupation number of the initial state for the JC case with $m=$ 1 (dashed line) and for $m=2$ (dotted line). The parameters for the plot on the left are $\eta=1.5, \Omega=0.5 \mathrm{GHz}, \nu=1.2 \omega_{0}=120 \mathrm{MHz}$, such that $\Phi_{1}^{1} \leq 0$ and $\Phi_{n}^{2} \geq 0, \forall n$. For the plot on the right, the parameters are $\eta=1.0, \Omega=1 \mathrm{GHz}, \nu=1.2 \omega_{0}=120 \mathrm{MHz}$, such that $\Phi_{1}^{1} \leq 0$ and $\Phi_{1}^{2} \leq 0$. The sum in Eq. (25) is truncated in $n=50$ terms [32].

example the carrier transition $(m=0)$ in Fig. 5 when, for a given trap frequency $\nu$, we vary $\eta$ from zero $(\phi=\pi / 2)$ to its maximum value $(\phi=0)$. This is repeated for a broad range of trap frequencies. In general, the effect of varying the frequency of the trap is just to limit the maximum attainable values of $\eta$ obtained by changing the laser propagation direction in relation to the trap axis (angle $\phi$ ). The NL basically does not change if $\nu$ is varied keeping $\eta$ fixed. Of course, according to Eq. (8), in order to keep $\eta$ fixed while changing $\nu$, the angle $\phi$ must also be varied. For $\nu \ll \omega_{0}(\nu \rightarrow 0)$ one can adjust $\phi$ in order to keep $\eta$ constant. This limit, obtained from Eq. (25), reads

$$
\frac{\mathcal{Z}_{ \pm}\left(\lambda_{f}\right)}{\mathcal{Z}\left(\lambda_{i}\right)} \rightarrow \operatorname{sech} \frac{\hbar \beta \omega_{0}}{2} \sum_{n=0}^{\infty} \cosh \left[\frac{\beta \hbar}{2} \sqrt{\omega_{0}^{2}+\Omega^{2}\left|f_{n}^{m}\right|^{2}}\right]
$$

regardless of being the JC or AJC case. Giving the convergence properties of $\left|f_{n}^{m}\right|$, discussed in [32], this limit is finite. This finite behavior is illustrated with the case $m=0$ in Fig. 5. Other choices of $m$ will lead to conclusions alike since the asymptotic behavior of $\left|f_{n}^{m}\right|$ with $n$ and $\eta$ does not depend on $m$ in any fundamental way [32].

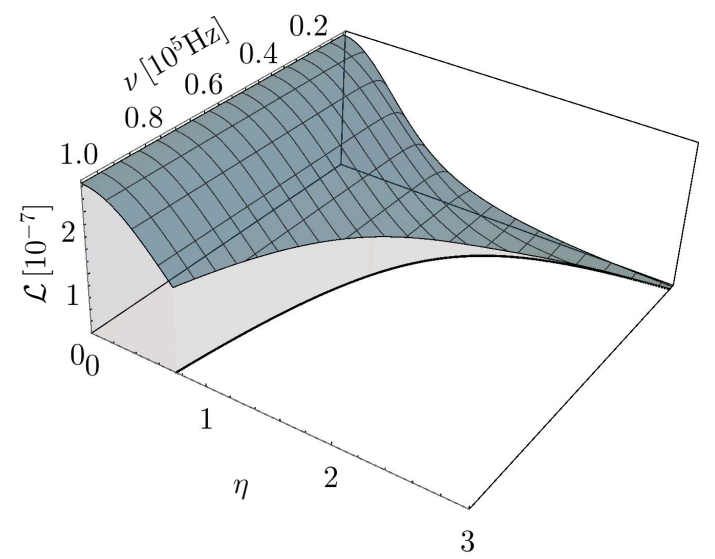

Figure 5. (Color Online) Nonequilibrium Lag as a function of the natural frequency for the case $m=0$. The solid plane curve is the projection of Eq. (8) with $\phi=0$ on the azimuthal plane $\mathcal{L}=$ $1.5 \times 10^{-7}$. The parameters for the plot are as in Fig. 1 . 
To finish the analysis of the NL, we explore its behavior for higher sidebands $(m>2)$. In Fig. 6, we present a numerical study of such a dependence. One can see that the JC case tends to reversibility as the number of excitations $m$ exchanged between the ion motion and the electronic levels, induced by the laser, increases. For the AJC case, once again a rich behavior is found. For small $\eta$, the NL monotonically decreases with $m$, while for higher values of $\eta$, it comes to a point where the behavior is not monotonic anymore as highlighted in the inset of the bottom panel in Fig. 6. From this point, we varied $\eta$ up to 3.5 (see Fig. 1) to verify that, in this range, the maximum displaces to higher values of $m$ as $\eta$ increases. The same kind of analysis was performed considering the variation of $m$ for different temperatures and Rabi frequencies, and contrary to results in Fig. 6, there are no remarkable differences between the AJC and JC cases.
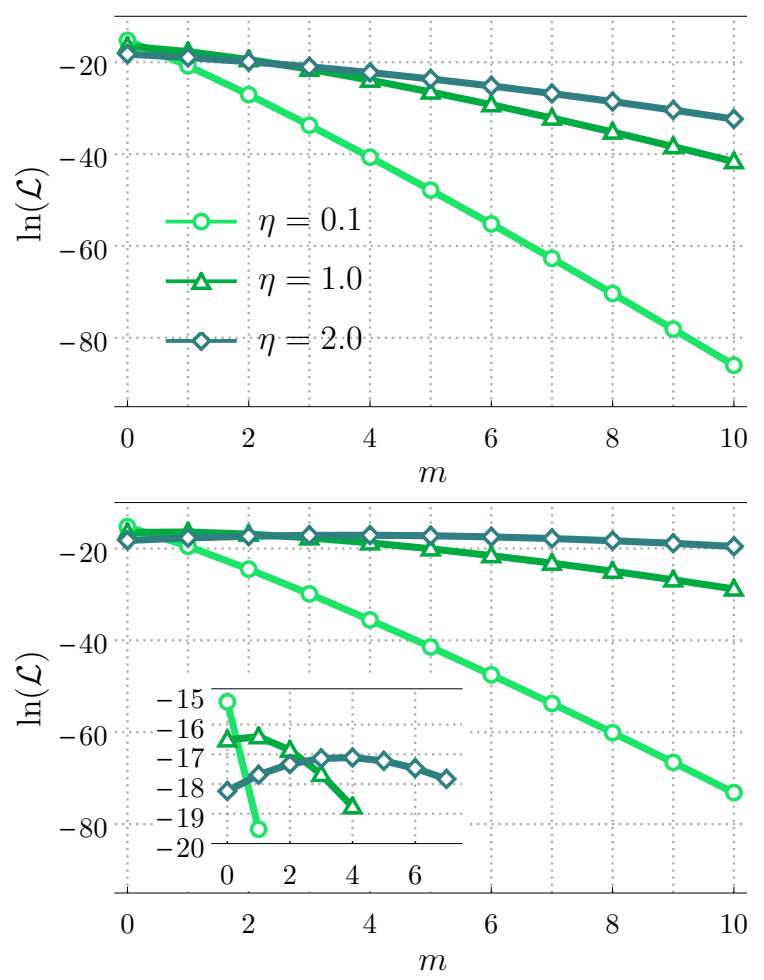

Figure 6. (Color Online) Nonequilibrium Lag as a function of the sideband number $m$ for some illustrative choices of the Lamb-Dicke parameter. Top: JC case. Bottom: AJC case. The parameters are the same as in Fig. 1.

As a final remark, it is worthwhile to notice that, except for Fig. 4, which is a theoretical extrapolation of the current experimental parameters, we have always found a higher NL for the AJC than for the JC. This can be once again understood from the relatively small values of $\bar{n}$ used in the simulations, and from the fact that the NL is a relative entropy. This is the same reasoning we employed in the analysis of Fig. 3. Additionally, the first order expansion of the hyperbolic function (in powers of $m \nu / \omega_{0}$ ) in Eq. (25) shows immediately that $\mathcal{Z}_{-}>\mathcal{Z}_{+}$.

\section{CONCLUSIONS}

From the point of view of non-equilibrium thermodynamics, we studied the problem of sudden driving of a trapped ion by a classical laser field. This thermodynamical analysis was instrumental to pinpoint fundamental differences between the Jaynes-Cummings and Anti-Jaynes-Cummings-type Hamiltonians that arise in the trapped ion system by careful choice of the laser frequency. The role played by the magnitude of the Lamb-Dicke parameter, related to nonlinearity, and other physically relevant parameters was carefully studied. This makes our work useful also to the experimentalist who might be interested in the practical investigation of quantum thermodynamics of laser-manipulated trapped ion systems. In this respect, our work is, to the best of our knowledge, the first one to include, in a thermodynamical approach, the great variety of possible electronic-vibration interactions available in the trapped ion system.

An experimental assessment of the findings of this paper might make use of a 2D trap (ion oscillations along $x$ and $y$ directions) and a driving laser coupling the electronic degrees of freedom to the $x$ motion. This can be easily achieved by choosing the right direction of the laser wave vector. The $y$ motion is used then as an ancilla in the interferometric scheme presented in [14]. For that, an extra laser is to be used to couple the system (electronic levels plus $x$ motion) to the ancilla in order to arrange for a proper gate entangling them [14].

\section{ACKNOWLEDGMENTS}

A.A.C. acknowledges to "Coordenação de Aperfeioamento de Pessoal de Nível Superior” (CAPES). FN, FLS and MP are supported by the CNPq "Ciência sem Fronteiras" programme through the "Pesquisador Visitante Especial" initiative (grant nr. 401265/2012-9). MP acknowledges financial support from John Templeton Foundation (grant ID 43467), the EU Collaborative Project TherMiQ (Grant Agreement 618074), and also gratefully acknowledge support from the COST Action MP1209 "Thermodynamics in the quantum regime". FLS is a member of the Brazilian National Institute of Science and Technology of Quantum Information (INCTIQ) and acknowledges partial support from CNPq (grant nr. 307774/2014-7).

\section{APPENDIX}

In this appendix we analytically perform the diagonalization of the Hamiltonians in Eq. (9) for any value of $m$.

\section{Diagonalization of $\hat{\mathcal{H}}_{+}^{(m)}$}

Let us consider the eigenbasis for the free Hamiltonian: $\{|n, e\rangle,|n, g\rangle ; n=0,1, \ldots, \infty\}$. It is easy to see that the subspace spanned by the set $\{|n, e\rangle,|n+m, g\rangle\}$ is invariant under 
the action of the JC like Hamiltonian, $\hat{\mathcal{H}}_{+}^{(m)}$, in Eq. (9) $\forall m, n$. Furthermore, if $m>n$ then it is true that

$$
\hat{\mathcal{H}}_{+}^{(m)}|n, g\rangle=\hat{\mathcal{H}}_{0}|n, g\rangle=\left(\hbar \nu n-\frac{\hbar \omega_{0}}{2}\right)|n, g\rangle
$$

i.e., the eigenstate $|n, g\rangle$ must be included in the invariant subspace, which becomes $\{|n, g\rangle,|n, e\rangle,|n+m, g\rangle\}$. Any matrix element of $\hat{\mathcal{H}}_{+}^{(m)}$ outside the invariant subspace is null because of (12).

Taking the matrix elements of the Hamiltonian in the invariant subspaces, and rearranging the basis, it acquires a simple block structure:

$$
\hat{\mathcal{H}}_{+}^{(m)}=\hat{\mathcal{H}}_{+}^{[1]} \oplus \hat{\mathcal{H}}_{+}^{[2]}
$$

with

$$
\begin{aligned}
\hat{\mathcal{H}}_{+}^{[1]} & =\bigoplus_{n=0}^{m-1}\left\langle n, g\left|\hat{\mathcal{H}}_{+}^{(m)}\right| n, g\right\rangle=\hbar \bigoplus_{n=0}^{m-1}\left(\nu n-\frac{\omega_{0}}{2}\right), \\
\hat{\mathcal{H}}_{+}^{[2]} & =\bigoplus_{n=0}^{\infty}\left(\begin{array}{cc}
\left\langle n, e\left|\hat{\mathcal{H}}_{+}^{(m)}\right| n, e\right\rangle & \left\langle n+m, g\left|\hat{\mathcal{H}}_{+}^{(m)}\right| n, e\right\rangle \\
\left\langle n, e\left|\hat{\mathcal{H}}_{+}^{(m)}\right| n+m, g\right\rangle\left\langle n+m, g\left|\hat{\mathcal{H}}_{+}^{(m)}\right| n+m, g\right\rangle
\end{array}\right) \\
& =\hbar \bigoplus_{n=0}^{\infty}\left(\begin{array}{cc}
\nu n+\frac{\omega_{0}}{2} & \mathrm{e}^{-i \omega_{L} t} \Omega f_{n}^{m} \\
e^{i \omega_{L} t} \Omega f_{n}^{m *} & \nu(n+m)-\frac{\omega_{0}}{2}
\end{array}\right),
\end{aligned}
$$

with $f_{n}^{m}$ defined in Eq. (26).

The above block structure enable us to diagonalize the Hamiltonian by the diagonalization of each block. The first $m$ blocks in Eq. (A-3) are matrices of only one element having eigenvalues and eigenvectors, respectively, given by

$$
\zeta_{+}^{(n, m)}=\hbar \nu n-\frac{\hbar \omega_{0}}{2},\left|\zeta_{+}^{(n, m)}\right\rangle=|n, g\rangle
$$

for each $n=0, \ldots, m-1$ for a given $m$. The following blocks in the diagonal block structure of (A-2) are $2 \times 2$ matrices, which can be diagonalized to give for all $n, m$ the eigenvalues

$$
\begin{aligned}
& \mu_{+}^{(n, m)}=\hbar \nu\left(n+\frac{m}{2}\right)-\frac{\hbar}{2} \sqrt{\omega_{L}^{2}+\Omega^{2}\left|f_{n}^{m}\right|^{2}}, \\
& \gamma_{+}^{(n, m)}=\hbar \nu\left(n+\frac{m}{2}\right)+\frac{\hbar}{2} \sqrt{\omega_{L}^{2}+\Omega^{2}\left|f_{n}^{m}\right|^{2}},
\end{aligned}
$$

respectively, associated to the eigenvectors

$$
\begin{aligned}
\left|\mu_{+}^{(n, m)}\right\rangle & =\frac{\mathrm{e}^{-i \omega_{L} t}\left[\omega_{L}-\sqrt{\omega_{L}^{2}+\Omega^{2}\left|f_{n}^{m}\right|^{2}}\right]}{\Omega f_{n}^{m *} \sqrt{1+\frac{\left|\omega_{L}-\sqrt{\omega_{L}^{2}+\Omega^{2}\left|f_{n}^{m}\right|^{2}}\right|^{2}}{\Omega^{2}\left|f_{n}^{m}\right|^{2}}}}|n, e\rangle \\
& +\frac{1}{\sqrt{1+\frac{\left|\omega_{L}-\sqrt{\omega_{L}^{2}+\Omega^{2}\left|f_{n}^{m}\right|^{2}}\right|^{2}}{\Omega^{2}\left|f_{n}^{m}\right|^{2}}}}|n+m, g\rangle, \\
\left|\gamma_{+}^{(n, m)}\right\rangle & =\frac{\mathrm{e}^{-i \omega_{L} t\left[\omega_{L}+\sqrt{\omega_{L}^{2}+\Omega^{2}\left|f_{n}^{m}\right|^{2}}\right]}}{\Omega f_{n}^{m *} \sqrt{1+\frac{\omega_{L}+\left.\sqrt{\omega_{L}^{2}+\Omega^{2}\left|f_{n}^{m}\right|^{2}}\right|^{2}}{\Omega^{2}\left|f_{n}^{m}\right|^{2}}}}|n, e\rangle \\
& +\frac{1}{\sqrt{1+\frac{\left|\omega_{L}+\sqrt{\omega_{L}^{2}+\Omega^{2}\left|f_{n}^{m}\right|^{2}}\right|^{2}}{\Omega^{2}\left|f_{n}^{m}\right|^{2}}}}|n+m, g\rangle,
\end{aligned}
$$

where in this regime $\omega_{L}=\left(\omega_{0}-m \nu\right)$.

2. Diagonalization of $\hat{\mathcal{H}}_{-}^{(m)}$

For the AJC like Hamiltonian, $\hat{\mathcal{H}}_{-}^{(m)}$, in Eq. (9), the invariant subspace is $\{|n+m, e\rangle,|n, g\rangle\}$ for all $m, n$, while for $m>n$ it should be replaced by $\{|n, e\rangle,|n+m, e\rangle,|n, g\rangle\}$. Taking the matrix elements of the Hamiltonian in these subspaces, and rearranging the basis as before, one finds

$$
\hat{\mathcal{H}}_{-}^{(m)}=\hat{\mathcal{H}}_{-}^{[1]} \oplus \hat{\mathcal{H}}_{-}^{[2]},
$$

where

$$
\begin{aligned}
& \hat{\mathcal{H}}_{-}^{[1]}=\bigoplus_{n=0}^{m-1}\left\langle n, e\left|\hat{\mathcal{H}}_{+}^{(m)}\right| n, e\right\rangle=\hbar \bigoplus_{n=0}^{m-1}\left(\nu n+\frac{\omega_{0}}{2}\right), \\
& \hat{\mathcal{H}}_{-}^{[2]}=\bigoplus_{n=0}^{\infty}\left(\begin{array}{cc}
\left\langle n, g\left|\hat{\mathcal{H}}_{+}^{(m)}\right| n, g\right\rangle & \left\langle n+m, e\left|\hat{\mathcal{H}}_{+}^{(m)}\right| n, g\right\rangle \\
\left\langle n, g\left|\hat{\mathcal{H}}_{+}^{(m)}\right| n+m, e\right\rangle\left\langle n+m, e\left|\hat{\mathcal{H}}_{+}^{(m)}\right| n+m, e\right\rangle
\end{array}\right) \\
&=\hbar \bigoplus_{n=0}^{\infty}\left(\begin{array}{cc}
\nu(n+m)+\frac{\omega_{0}}{2} & \mathrm{e}^{-i \omega_{L} t} \Omega f_{n}^{m} \\
\mathrm{e}^{i \omega_{L} t} \Omega f_{n}^{m *} & \nu n-\frac{\omega_{0}}{2}
\end{array}\right),
\end{aligned}
$$

and $f_{n}^{m}$ is defined in Eq. (26).

Now considering the one dimensional blocks where $m>n$, its eigenvalues and eigenvectors are, respectively, given by

$$
\zeta_{-}^{(n, m)}=\hbar \nu n+\frac{\hbar \omega_{0}}{2},\left|\zeta_{-}^{(n, m)}\right\rangle=|n, e\rangle,
$$

for each $n=0, \ldots, m-1$ for a given $m$. The eigenvalues of each $2 \times 2$ blocks in Eq. (A-8) now becomes

$$
\begin{aligned}
& \mu_{-}^{(n, m)}=\hbar \nu\left(n+\frac{m}{2}\right)-\frac{\hbar}{2} \sqrt{\omega_{L}^{2}+\Omega^{2}\left|f_{n}^{m}\right|^{2}} \\
& \gamma_{-}^{(n, m)}=\hbar \nu\left(n+\frac{m}{2}\right)+\frac{\hbar}{2} \sqrt{\omega_{L}^{2}+\Omega^{2}\left|f_{n}^{m}\right|^{2}},
\end{aligned}
$$

respectively, associated to the eigenvectors

$$
\begin{aligned}
\left|\mu_{-}^{(n, m)}\right\rangle & =\frac{\mathrm{e}^{-i \omega_{L} t}\left[\omega_{L}-\sqrt{\omega_{L}^{2}+\Omega^{2}\left|f_{n}^{m}\right|^{2}}\right]}{\Omega f_{n}^{m *} \sqrt{1+\frac{\omega_{L}-\sqrt{\omega_{L}^{2}+\Omega^{2}\left|f_{n}^{m}\right|^{2}}}{\Omega^{2}\left|f_{n}^{m}\right|^{2}}}}|n, g\rangle \\
& +\frac{1}{\sqrt{1+\frac{\left|\omega_{L}-\sqrt{\omega_{L}^{2}+\Omega^{2}\left|f_{n}^{m}\right|^{2}}\right|^{2}}{\Omega^{2}\left|f_{n}^{m}\right|^{2}}}}|n+m, e\rangle, \\
\left|\gamma_{-}^{(n, m)}\right\rangle & =\frac{\mathrm{e}^{-i \omega_{L} t}\left[\omega_{L}+\sqrt{\omega_{L}^{2}+\Omega^{2}\left|f_{n}^{m}\right|^{2}}\right]}{\Omega f_{n}^{m *} \sqrt{1+\frac{\omega_{L}+\sqrt{\omega_{L}^{2}+\Omega^{2}\left|f_{n}^{m}\right|^{2}}}{\Omega^{2}\left|f_{n}^{m}\right|^{2}}}}|n, g\rangle \\
& +\frac{1}{\sqrt{1+\frac{\left|\omega_{L}+\sqrt{\omega_{L}^{2}+\Omega^{2}\left|f_{n}^{m}\right|^{2}}\right|^{2}}{\Omega^{2}\left|f_{n}^{m}\right|^{2}}}}|n+m, e\rangle,
\end{aligned}
$$

for all $n, m$ and in this regime $\omega_{L}=\left(\omega_{0}+m \nu\right)$.

As a final comment, the carrier transitions, Eq.(11), eigenvalues can be obtained either from Eq. (A-5) or from Eq. (A10), as its corresponding eigenvectors from Eq. (A-6) or Eq. (A-11) just setting $m=0$. 
[1] H. Rabitz, Focus on Quantum Control, New J. Phys. 11, 105031 (2009).

[2] D. Leibfried, R. Blatt, C. Monroe, and D. Wineland, Quantum dynamics of single trapped ions, Rev. Mod. Phys. 75, 281 (2003).

[3] R. Blatt and D. Wineland, Entangled states of trapped atomic ions, Nature 453, 1008 (2008).

[4] Ch. Roos et al., Quantum State Engineering on an Optical Transition and Decoherence in a Paul Trap, Phys. Rev. Lett. 83, 4713 (1999);

[5] D.M. Meekhof et al., Generation of Nonclassical Motional States of a Trapped Atom, Phys. Rev. Lett. 76, 1796 (1996); F. Schmidt-Kaler et al., How to realize a universal quantum gate with trapped ions, Appl. Phys. B 77, 789 (2003).

[6] F. Plastina et al., Irreversible Work and Inner Friction in Quantum Thermodynamic Processes, Phys. Rev. Lett 113, 260601 (2014).

[7] P. Talkner, E. Lutz, and P. Hänggi, Fluctuation theorems: Work is not an observable, Phys. Rev. E 75, 50 (2007); M. Esposito, U. Harbola, and S. Mukamel, Nonequilibrium fluctuations, fluctuation theorems, and counting statistics in quantum systems, Rev. Mod. Phys. 81, 1665 (2009).

[8] M. Campisi, P. Hänggi, and P. Talkner, Colloquium: Quantum fluctuation relations: Foundations and applications, Rev. Mod. Phys. 83, 771 (2011).

[9] C. Jarzynski, Nonequilibrium Equality for Free Energy Differences, Phys. Rev. Lett. 78, 2690 (1997).

[10] G.E. Crooks, Entropy production fluctuation theorem and the nonequilibrium work relation for free energy differences, Phys. Rev. E 60, 2721 (1999).

[11] H. Tasaki, Jarzynski Relations for Quantum Systems and Some Applications, arXiv:cond-mat/0009244v2 [cond-mat.statmech] (2000).

[12] R. Klages, W. Just, and C. Jarzynski, Nonequilibrium Statistical Physics of Small Systems: Fluctuation Relations and Beyond (Wiley-VCH Verlag \& Co. KGaA, Boschstr, 2013); C. Bustamante, J. Liphardt, and F. Ritort, The Nonequilibrium Thermodynamics of Small Systems, Physics Today 58, 43 (2005); J. Gemmer, M. Michel, and G. Mahler, Quantum Thermodynamics, Emergence of Thermodynamic Behavior Within Composite Quantum Systems (2 ${ }^{\text {nd }}$ Ed., Springer, Berlin, 2010).

[13] M. Campisi, Fluctuation Relation for Quantum Heat Engines and Refrigerators, J. Phys. A: Math. Theor. 47, 245001 (2014); M. Campisi, J. Pekola, and R. Fazio, Nonequilibrium Fluctuations in Quantum Heat Engines: Theory, Example, and Possible Solid State Experiments, New Journal of Physics 17, 035012 (2015); T.D. Kieu, Quantum Heat Engines, The Second Law and Maxwells Daemon, The European Physical Journal D 39, 115 (2006); V. Blickle and C. Bechinger, Realization of a micrometre-sized stochastic heat engine, Nature Physics 8, 143 (2011).

[14] T.B. Batalhão et al., Experimental Reconstruction of Work Distribution and Study of Fluctuation Relations in a Closed Quantum System, Phys. Rev. Lett. 113, 140601 (2014); R. Dorner et al., Extracting Quantum Work Statistics and Fluctuation Theorems by Single-Qubit Interferometry, Phys. Rev. Lett. 110, 230601 (2013); L. Mazzola, G. De Chiara, and M. Paternostro, Measuring the Characteristic Function of the Work Distribution, Phys. Rev. Lett. 110, 230602 (2013).

[15] M. Campisi, P. Talkner, and P. Hänggi, Fluctuation Theorem for Arbitrary Open Quantum Systems, Phys. Rev. Lett. 102, 210 401 (2009); P. Talkner, M. Campisi, and P. Hänggi, Fluctuation Theorems in Driven Open Quantum Systems, J. Stat. Mec. 2009 02025 (2009).

[16] A. Carlisle et al., Out of equilibrium thermodynamics of quantum harmonic chains, ArXiv:1403.0629 [quant-ph] (2014).

[17] M. Orszag, Quantum Optics (2 ${ }^{\text {nd }}$ Ed., Springer-Verlag, Berlin, 2008).

[18] O. Abah et al., Single-Ion Heat Engine at Maximum Power, Phys. Rev. Lett. 109, 203006 (2012); J. Roßnagell et al., A single-atom heat engine, Science 352, 325 (2016).

[19] S. An et al., Experimental Test of the Quantum Jarzynski Equality with a Trapped-Ion System, Nat. Phys. 11, 193 (2014); G. Huber, F. Schmidt-Kaler, S. Deffner, and E. Lutz, Employing Trapped Cold Ions to Verify the Quantum Jarzynski Equality, Phys. Rev. Lett. 101, 070403 (2008).

[20] S. Deffner and E. Lutz, Generalized Clausius Inequality for Nonequilibrium Quantum Processes, Phys. Rev. Lett. 105, 170402 (2010).

[21] R. Balian, From Microphysics to Macrophysics: Methods and Applications of Statistical Physics, (Vol I, Springer, Berlin 2007).

[22] L. Fusco et al., Assessing the Nonequilibrium Thermodynamics in a Quenched Quantum Many-Body System via Single Projective Measurements, Phys. Rev. X 4, 031029 (2014).

[23] W.L. Ribeiro, G.T. Landi, and F.L. Semião, Non-equilibrium thermodynamics of magnetic resonance using the quantum mechanics, ArXiv:1601.01833 [quant-ph] (2016).

[24] V. Vedral, The Role of Relative Entropy in Quantum Information Theory, Rev. Mod. Phys. 74, 197 (2002).

[25] P.K. Ghosh, Ion Traps (Oxford University Press, New York, 1995).

[26] C.A. Blockley, D.F. Walls and H. Risken, Quantum Collapses and Revivals in a Quantized Trap, Europhys. Lett. 17 (6), 509 (1992).

[27] D.J. Wineland et al., Experimental Issues in Coherent Quantum-State Manipulation of Trapped Atomic Ions, J. Res. Natl. Inst. Stand. Technol. 103, 259 (1998).

[28] I.S. Gradshteyn and I.M. Ryzhik, Table of Integrals, Series, and Products ( $7^{\text {th }}$ Ed, Elsevier, Amsterdam, 2007).

[29] W. Vogel and D.-G. Welsch, k-photon Jaynes-Cummings model with coherent atomic preparation: Squeezing and coherence, Phys. Rev. A 40, 7113 (1989).

[30] W. Vogel and R. L. de Matos Filho, Nonlinear Jaynes-Cummings dynamics of a trapped ion, Phys. Rev. A 52, 4214 (1995).

[31] S. Olmschenk et al., Manipulation and detection of a trapped $\mathrm{Yb}^{+}$hyperfine qubit, Phys. Rev. A 76, 052314 (2007).

[32] Numerical investigation of the sum in Eq. (25) shows that it is convergent since $\left|f_{n}^{m}\right|$, defined by Eq. (26), is a decreasing and oscillating function of $\eta$ and $n$. Some analytic progress is also possible to be made for large $n$ giving the well known asymptotic limit of Laguerre polynomials [28] appearing in Eq. (26). It can be shown that $\left|f_{n}^{m}\right| \sim n^{-1 / 4} \eta^{-1 / 2}$ in accordance with the tendency with $\eta$ and $n$ numerically revealed. The sum is truncated as soon as the relative difference between successive terms become about $10^{-10}$. In this way, the number of terms kept in the sum may vary in different plots as it is clearly depends on the specific set of parameters used to produce the plot. We indicate the truncation number in the caption of each figure. 